\title{
Prompted versus trial-and-error initial object discrimination learning by monkeys, retardates, and preschool children'
}

\author{
Harold J. Fleteher, J. Kent Davis, Bill M. Orr and Patricia Ross \\ UNIVERSITY OF WISCONSIN
}

\begin{abstract}
Abstraet
Following pretraining to a prompt (a light cue) naive monkeys, retardates, and preschool children were tested on 2 36-trial object discrimination problems under either a conventional trial-and-error condition or one involving prompting for the first 6 trials. While monkeys' performance on trials 7-36 was equivalent for both conditions, human Ss learned more during 6 prompted trials than during either the same number of conventional trials or during as many conventional trials as were needed to make 6 correct responses.
\end{abstract}

\section{Problem}

Guided learning, an old problem in psychology, is enjoying renewed interest (e. g., Cook \& Spitzer, 1960; Cochrane \& Davis, 1961; Hawker, 1964), and organismic and situational determinants of the relative effectiveness of various procedures are being identified. Examined here is the effect of prompting on discrimination learning by three types of primates.

\section{Method}

From among populations of Ss, naive with respect to object discrimination learning, the following completed the experiment: 20 severely retarded males with various etiologies from Central Wisconsin Colony (MA's 23-67 mos., median 44); six male and six female children from a local preschool nursery (ages 48-65 mos., median 52); and six adolescent feral female rhesus monkeys from the University of Wisconsin Primate Laboratory.

Retardates and children were tested in a modified WGTA in which $\mathrm{E}$ sat behind a one-way mirror, raised a door below the mirror, and pushed a presentation tray toward S. The tray, made completely from opaque white acrylic plastic, contained two channels in which slid $4 \times 4$ in white plastic bases. In their forward position the bases butted against the front edge and covered two foodwells 10 in apart. In the tray's front edge, 2-1/2 in high and angled $45^{\circ}$ from horizontal, were two centered 1 in jeweled amber lights (prompts) directly in front of the foodwells. Multidimensional junk objects were attached to the bases, and $S$ was required to push the object back to expose a foodwell. Monkeys were tested in a conventional WGTA with the same tray and stimuli. Raisin rewards were used for all Ss.

\section{Pretraining Phase}

All Ss were trained to displace a single 3 x 3 in gray block to obtain a reward. (For human Ss verbal instructions were given with regard to the requirements of the response.) When each $\mathrm{S}$ responded appropriately,
20 trials were given during which the single gray block appeared randomly on each side and the prompt (amber light) was lighted on that side. On the following day each $\mathrm{S}$ was given 36 trials during which two identical gray blocks appeared with the prompt indicating the rewarded block. (Verbal instructions during this and all subsequent phases were limited to essentially "find the raisin." No explanation of the significance of the prompt was offered.) All Ss were given 36 trials daily on this phase until a criterion of 30 correct responses within a session was met. The noncorrection procedure was employed in this and all subsequent phases, and rewarded position was randomized but balanced over blocks of six trials. To provide additional reinforcement for responding appropriately to the prompt and to familiarize Ss with junk objects, three different pairs of identical objects were presented, one pair each day for 36 trials, with the prompt lighted in front of the rewarded object. (Because of the ease with which the children met the criterion with the two gray blocks, these three pairs were all presented within a single session with 12 trials per pair.)

\section{Discrimination Learning Phase}

On the day following successful completion of pretraining Ss began the test phase which consisted of two 36-trial object discrimination problems, one problem per day, one under a prompted (P) and one under a trial-and-error $(\mathrm{T})$ condition. The $\mathrm{P}$ condition involved the presence of the light in front of the correct stimulus during each of the first six trials only; trials 7-36 followed without interruption but without the prompt. The $\mathrm{T}$ condition consisted of 36 conventional trials (no prompt). In order to balance and assess a possible order effect, Ss were randomly assigned to two groups which differed only with respect to the order in which the two conditions were given. For each $\mathrm{S}$ independently the four stimuli were randomly selected from among six objects.

\section{Results and Diseussion}

Performance in the presence of the prompt indicated that it was effective but differentially so. Monkeys, retardates, and children averaged, respectively, 86, 97 , and $100 \%$ correct on all prompted discrimination trials.

The main dependent variable, number of correct responses on trials 7-36, was subjected to a $2 \times 2$ replicated Latin Square analysis of variance which 


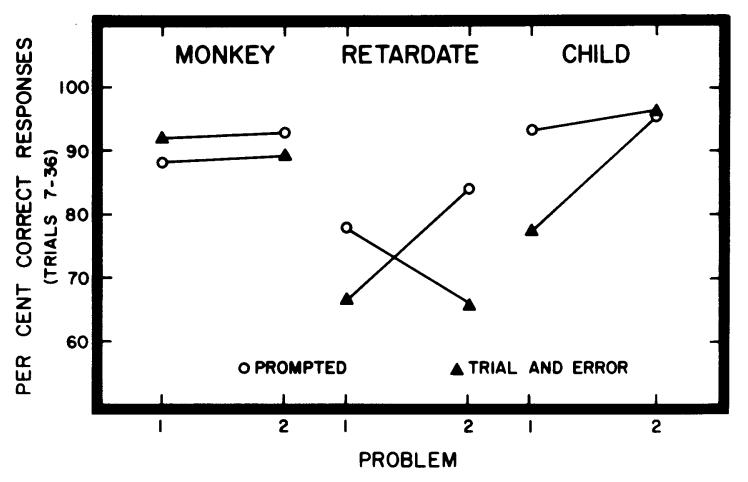

permitted independent evaluations of the effects of Conditions, Groups, and Problems (first or second). (On Problem 2 under the $\mathrm{T}$ condition one retardate made 36 errors, an obvious stimulus preference, so his score was replaced by the cell mean.) As may be inferred from inspection of the figure, none of these effects was significant (all $\mathrm{F}^{\prime} \mathrm{s}<1.0$ ) for monkeys which solved these relatively easy discriminations with equal facility under both conditions. The retardates, however, profited significantly more from six prompted trials than they did from six conventional trials on both problems $(F=13.97 ; d f=1 / 17 ; p<.01)$; no other effects were significant. Analyses of the children's data allowed the same inference, but although the main effect of conditions was significant $(F=10.81 ; \mathrm{df}=1 / 10 ; \mathrm{p}<.01)$, the superiority of prompting was obviously restricted to the first problem. The main effect of problems was also significant $(F=18.36 ; \mathrm{df}=1 / 10 ; \mathrm{p}<.01)$. In summary, retardates (both problems) and children (first problem only) learned more during six prompted trials than during six conventional discrimination trials.

A speciously simple interpretation would attribute the superiority of prompting to the fact that more correct instrumental responses necessarily occurred during prompted trials, hence greater habit strength was established during these trials than during six conventional trials during which fewer correct responses occurred. This interpretation was rejected by the following analysis.

For each $\mathrm{S}$ under the $\mathrm{T}$ condition six correct responses were counted, and the per cent of correct responses on all remaining trials was determined. These scores were compared to the per cent of correct responses on the 30 trials following the prompted trials for a test of the hypothesis that performance following six rewarded responses should be at le a $\mathrm{s} t$ as good as performance following six prompted trials. (Indeed some theorists would argue that the effects of the incorrect responses, added to the effects of six rewarded responses, should produce superior performance.) Over both problems retardates averaged $80 \%$ following prompting and only $69 \%$ following six correct trial-anderror responses. (Of 18 untied scores only four were higher following the six correct responses; $p=.015$.) Similarly, but on the first problem only, children averaged $93 \%$ correct following prompting and only $82 \%$ following six correct responses. (A Mann-Whitney test supported the apparent superiority of the P condition; $\mathrm{U}=7 ; \mathrm{p}=.053$.) Thus in each instance in which prompting was relatively better, there remained a reliable $11 \%$ accuracy which could not be attributed to reinforcement of an equivalent number of overt responses.

An alternative interpretation requires a reconsideration of events which presumably occur during a prompted trial. The prompt essentially elicits some form of an implicit response sequence $(r)$, which necessarily involves the prompted object, and which is normally followed by the explicit instrumental response (R) to that object. On the basis of the present data, it is suggested that $r$ functions similarly to, but independently of, $R, i$. e., the sheer occurrence of the elicited implicit response sequence increases the tendency to respond explicitly to the object involved, and the strength of this tendency is independent of the same tendency which increases as a function of rewarded instrumental responses. This interpretation, then, attributes the superiority of the $\mathrm{P}$ condition to the occurrence of two events ( $r$ plus $R$ ), each of which contributes in d e p e n dently to the strength of the correct response. The $T$ condition obviously involves only the single event (R) on each trial, and therefore the probability of a subsequent correct response is some finite amount less.

\section{References}

COCHRANE, R., \& DAVIS, R. T. Guided-choice training of young monkeỳs. Psychol. Rep., 1961, 9, 223-226.

COOK, J. O., \& SPITZER, M. E. Prompting versus confirmation in paired-associate learning. J. exp. Psychol., 1960, 59, 275-276.

HAWKER, J. R. Training procedure and practice-test ratio in the acquisition and retention of a serial maze pattern. Psychon. Sci., 1964, 1, 97-98.

\section{Note}

1. This research was supported in part by the Wisconsin Alumni Research Foundation. The authors thank Mr. C. F. Zmudzinski, director of the Madison Neighborhood House daytime nursery program, for his kind cooperation. 